Case Report

\title{
Pemphigus, discoid lupus erythematosus, and dermatomyositis during an 8-year follow-up period: a case report
}

\author{
Kobkan Thongprasom 1), Suwan Prasongtanskul2), Achara Fongkhum³), \\ and Anak Iamaroon ${ }^{4)}$
1)Department of Oral Medicine, Faculty of Dentistry, Chulalongkorn University, Bangkok, Thailand
2)Dental Division, Lamphun Hospital, Lampun, Thailand
${ }^{3)}$ Department of Internal Medicine, Lamphun Hospital, Lamphun, Thailand Chiang Mai, Thailand \\ 4)Department of Oral Biology and Diagnostic Sciences, Faculty of Dentistry, Chiang Mai University,
}

(Received February 9, 2013; Accepted May 31, 2013)

\begin{abstract}
A 36-year-old Thai woman presented with a painful, burning sensation in the gingiva and desquamative gingivitis. Findings from histopathologic and direct immunofluorescence examination of the maxillary gingiva confirmed a diagnosis of pemphigus. Two months later, she developed a round erythematous lesion on her face, and findings from a skin biopsy were consistent with lupus erythematosus. Four years after initial presentation, the patient developed amyotrophic dermatomyositis. Seven years after initial presentation, she developed joint pain and tenositis of the neck, shoulders, knees and ankles, which was diagnosed as asymmetric polyarthritis and multiple enthesopathy. There are no previous reports of pemphigus, discoid lupus erythematosus, and dermatomyositis in a Thai patient. Fluocinolone acetonide $0.1 \%$ in Orabase and systemic steroid were effective in treating oral $\mathrm{PV}$ in this patient. The gingiva showed complete remission after 10 months of treatment and remission with recession at 8.5 years of follow-up. (J Oral Sci 55, 255-258, 2013)
\end{abstract}

Keywords: dermatomyositis; enthesopathy; fluocinolone acetonide; lupus erythematosus; pemphigus vulgaris; oral.

Correspondence to Dr. Kobkan Thongprasom, Department of Oral Medicine, Faculty of Dentistry, Chulalongkorn University, Bangkok 10330, Thailand

Fax:+66-2-2188941 E-mail: kobkan.t@chula.ac.th

\section{Introduction}

Oral pemphigus vulgaris (PV) is a vesiculobullous disease and is potentially fatal if untreated. Oral manifestations usually precede skin lesions (1). PV is believed to be a rare autoimmune disease among Thais (2). Although the cause of PV is not known, it is important that treatment be given to avoid progression to skin lesions. While a few cases of PV with discoid lupus erythematosus (DLE) and dermatomyositis have been reported, none involved Thais (3-6). The pathogenesis of dermatomyositis is poorly understood. It is classified as an idiopathic inflammatory myopathy and is characterized by cutaneous manifestations, mostly in skin and muscle. Amyotrophic dermatomyositis is the term used to describe the small subgroup of patients that have a skin rash typical of dermatomyositis but no muscle involvement (7). Early diagnosis is useful in successfully controlling oral lesions. Here we describe a rare case of PV, DLE, and dermatomyositis, which occurred during a follow-up period of 8.5 years.

\section{Case Report}

A 36-year-old Thai woman presented with a 2-week history of generalized pain, and burning sensation, in the gingiva and bleeding during toothbrushing. Her dentist had treated the gingival lesions with scaling and root planing, but the lesions showed no sign of improvement. Intraoral examination revealed generalized erythema of the marginal gingiva and bilateral erosive, desquamative epithelium with slough on the interdental and marginal 


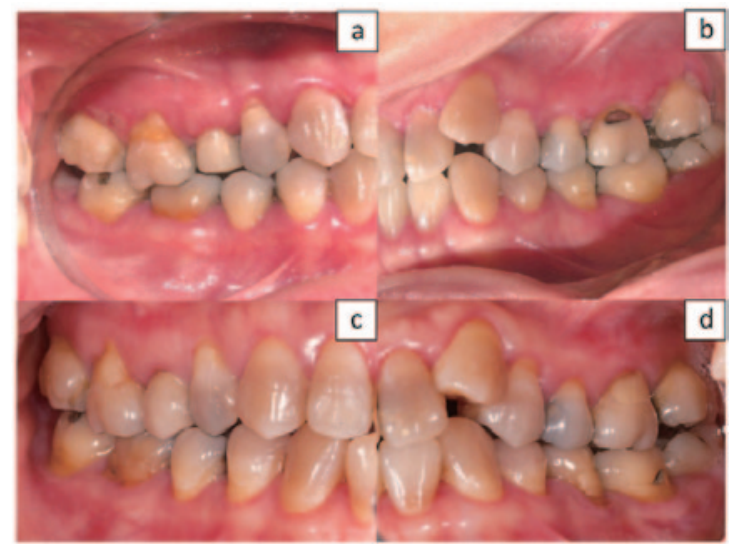

Fig. 1 Erosive, desquamative gingivitis with slough on interdental and marginal papillae at right (a) and left (b) maxillary second premolars to second molars. The gingiva showed complete remission with recession at 8.5 years of follow-up (c) and (d).

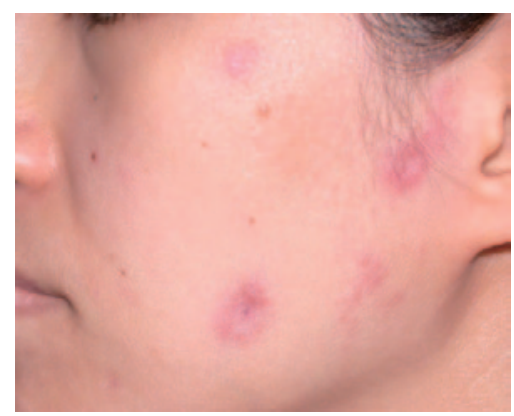

Fig. 3 Erythematous facial rash with acne-like papules.

papillae of the maxillary second premolars and first molars (Fig. 1a, b). Small amounts of dental plaque and calculus were observed in these areas. An incisional biopsy of the maxillary gingiva was performed for histopathologic and direct immunofluorescence examination. Histopathologic findings revealed acantholysis of the epithelial cells, which exhibited intraepithelial separation, particularly in the lower spinous cell layer, and perivascular infiltration of lymphocytes in the lamina propria (Fig. 2). Direct immunofluorescence revealed IgG deposition at intercellular bridges between epithelial cells; however, a direct immunofluorescence photomicrograph was not obtained at that time. Taken together, these findings were consistent with a final diagnosis of oral pemphigus vulgaris (PV). Two months later, the patient developed an erythematous facial rash with acnelike papules (Fig. 3), and the findings of a subsequent skin biopsy were consistent with lupus erythematosus (LE).

Complete blood counts were all normal. A Venereal Disease Research Laboratory (VDRL) test was nonreactive with LE cells, and the antinuclear antibody was also negative. Her complement level was normal, as

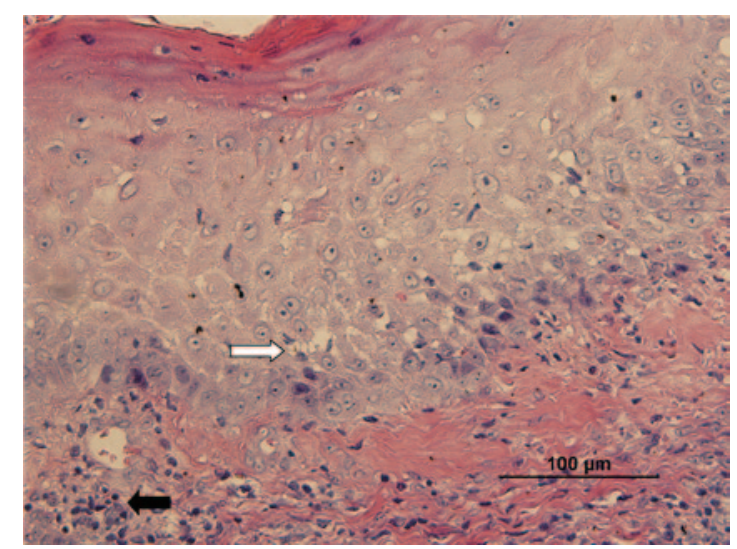

Fig. 2 Histopathologic examination showed intraepithelial separation, particularly at the lower spinous cell layer (white arrow), and perivascular infiltration of lymphocytes in the lamina propria (black arrow).

was her serum creatine phosphokinase (CPK) level. Initial laboratory investigations for antinuclear antibody (ANA), LE, rheumatoid factor, anti-double-stranded DNA, anti-Ro, anti-La, anti-smith, and anti-RNP were negative. However, ANA was weakly positive and showed a diffuse coarse speckled pattern. Liver function tests, blood urea nitrogen, creatinine, thyroid function tests, and erythrocyte sedimentation rate were all normal (Table 1). Repeated malignancy screens were negative. Ultrasonography revealed right and left ovarian cysts.

The patient was started on $30 \mathrm{mg} /$ day oral prednisolone, and the oral lesions were treated with fluocinolone acetonide $0.1 \%$ in Orabase (FAO $0.1 \%$ ). After 2 weeks of treatment, the lesions had gradually improved, and nearly complete remission was achieved at 10 months. One month after treatment, an erythematous rash, with alopecia areata, developed on her scalp. She was treated with dapsone, but drug-induced hemolysis occurred at 2 weeks of treatment.

The patient was treated with many medications, including continuous $20-30 \mathrm{mg} /$ day oral prednisolone. FAO $0.1 \%$ was applied to the gingiva until disease remission was confirmed. She was also treated with chloroquine, methotrexate, colchicine, and celebrex (Table 2).

One year later, she experienced a flare-up of the skin lesion: a photosensitive rash developed on her face (malar rash) and on a V-shaped area around the neck. Four years later, she developed erythematous desquamation of the fingers ("mechanic's hands") (Fig. 4a) and periungual telangiectasia. Gottron papules were noted at the interphalangeal joints (Fig. 4b).

Amyotrophic dermatomyositis was diagnosed. Interestingly, 7 years after initial presentation we noted 
Table 1 Results of blood tests

\begin{tabular}{lll}
\hline Blood test & Results & Remarks \\
\hline Complete blood count & All normal & Normal \\
Venereal Disease Research Laboratory test & Nonreactive & - \\
Antinuclear antibody & Negative & Weak; diffuse coarse speckled pattern \\
Lupus erythematosus cells & Negative & - \\
Creatine phosphokinase & $30-70 \mathrm{U} / \mathrm{L}$ (range) & Normal range in females $=29-168 \mathrm{U} / \mathrm{L}$ \\
Erythrocyte sedimentation rate & $30-50 \mathrm{~mm} / \mathrm{h}($ range) & Normal to slightly high \\
& & Normal range in females $=0-20 \mathrm{~mm} / \mathrm{h}$ \\
Rheumatoid factor & Negative & - \\
Anti-double-stranded DNA & Negative & - \\
Anti-Ro, Anti-La, Anti-Sm & Negative & - \\
Anti-ribonucleoprotein & Negative & - \\
C3 & $129 \mathrm{mg} / \mathrm{dL}$ & Normal $(83-172 \mathrm{mg} / \mathrm{dL})$ \\
CH50 & $182 \mathrm{U} / \mathrm{mL}$ & Normal $(142-279 \mathrm{U} / \mathrm{mL})$ \\
\hline
\end{tabular}

Table 2 Drugs used in treatment

\begin{tabular}{|c|c|c|c|}
\hline Medications & Prescription & Duration & Remarks \\
\hline Oral prednisolone (5 mg) & $\begin{array}{l}30 \mathrm{mg} / \text { day } \\
20 \mathrm{mg} / \text { day } \\
10 \mathrm{mg} / \text { day } \\
10 \mathrm{mg} / \mathrm{AD}\end{array}$ & $\begin{array}{l}4 \text { months } \\
2 \text { months } \\
2 \text { months } \\
2 \text { months }\end{array}$ & $\begin{array}{l}\text { Good response in intraoral lesion; no } \\
\text { response in skin lesion }\end{array}$ \\
\hline $\begin{array}{l}\text { Fluocinolone acetonide } 0.1 \% \text { Orabase } \\
\text { ( } 5 \mathrm{mg} / \text { pack) }\end{array}$ & tid pc. & 10 months & $\begin{array}{l}\text { Gradual improvement after } 2 \text { weeks; } \\
\text { complete remission at } 10 \text { months }\end{array}$ \\
\hline Doxycycline (100 mg) & 2 tabs/day & 1 month & No response in skin lesion \\
\hline Dapsone (100 mg) & $100 \mathrm{mg} /$ day & 2 weeks & $\begin{array}{l}\text { Drug-induced hemolysis occurred } \\
\text { after } 2 \text { weeks of treatment }\end{array}$ \\
\hline Chloroquine (250 mg) & $250 \mathrm{mg} /$ day & 2 years & No response in skin lesion \\
\hline Methotrexate (2.5 mg) & 3 tabs/day & 4 months & No response in skin lesion \\
\hline Colchicine (0.6 mg) & $1 \mathrm{tab} /$ day & 1 month & No response in skin lesion \\
\hline Celebrex (200 mg) & $1 \mathrm{tab} /$ day & $\begin{array}{l}4 \text { months; then as } \\
\text { needed for pain }\end{array}$ & $\begin{array}{l}\text { For treatment of enthesopathy and } \\
\text { arthritis at year } 7 \text { of follow-up }\end{array}$ \\
\hline
\end{tabular}

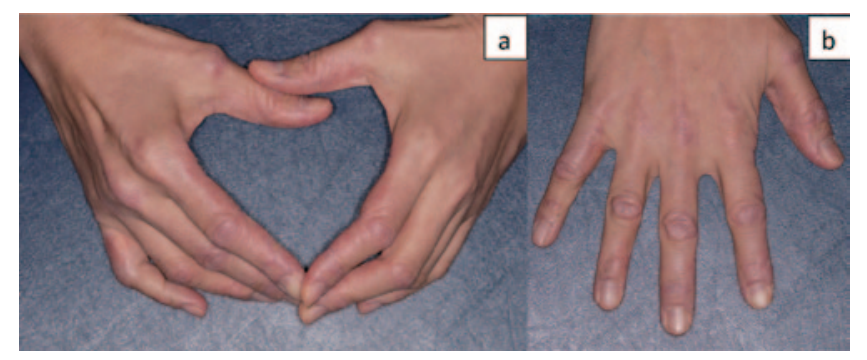

Fig. 4 Erythematous desquamation ("mechanic's hands") (a) and interphalangeal joints with Gottron papules (b).

gingival recession, although the oral lesions had completely resolved. In addition, she reported joint pain at the knees, ankles, shoulders, and neck and had developed asymmetric polyarthritis and multiple enthesopathy.

Due to her use of many prescription medications, she experienced numerous side- effects, which she ultimately could not tolerate. As a result, she elected to stop using all medications. She sought alternative treatments, including dharma meditation therapy, relaxation, and stress reduction, and attempted natural therapies, including avoidance of hot food, spicy food, and meat. At 8.5 years of follow-up, her gingiva remains in remission, although with some gingival recession (Fig. 1c, d); however, the facial lesions have only partially resolved. In sum, her condition and quality- of- life have improved.

\section{Discussion}

Oral PV is a vesiculobullous disease in which oral manifestations usually precede skin lesions. It is classified as an autoimmune disease and is potentially fatal if untreated. PV is believed to be rare in Thais, and most patients with PV present with gingival lesions-desquamative epithelium in 17 of 18 cases (94\%) in one study (2). In our patient, the maxilla gingiva showed erythematous changes, with desquamative epithelium. Histopathologic study of a gingival specimen showed parakeratinized stratified squamous epithelium with acanthosis, and loss of cellular cohesion in basal and spinous cells, suggesting intraepithelial vesicular formation. The underlying 
fibrous connective tissue was well vascularized and had severe chronic inflammatory infiltrate. Moreover, immunostaining showed IgG-positivity in the intercellular spaces of epithelium from the gingival specimen. These findings helped confirm a diagnosis of PV.

Histopathologic examination and analysis of a biopsy specimen of the round lesions on her face revealed mild vascular degeneration of epidermal basal cells and follicular plugging. The basement membrane appeared normal. Mononuclear perivascular infiltrate consisting mostly of lymphocytes was noted in the superficial and deep dermal layers and in subcutaneous tissue. Infiltration was also seen around skin appendages and in peripheral nerve fibers. The clinical presentation and histopathologic results were consistent with lupus erythematosus.

This patient had photosensitive areas, scalp lesions, malar rash, Gottron papules, and mechanic's hands, which met the criteria for a diagnosis of amyotrophic dermatomyositis. Seven years after initial presentation, she developed joint pain and tenositis of the neck, shoulders, knees and ankles; thus, the ultimate diagnosis was asymmetric polyarthritis and multiple enthesopathy.

The patient had been treated with many medications, including continuous $20-30 \mathrm{mg} /$ day oral prednisolone; FAO $0.1 \%$ was applied to the gingiva until disease remission. The gingiva showed complete remission after treatment for 10 months and remission with recession at 8.5 years of follow-up. Chloroquine, methotrexate, colchicine, and celebrex were also prescribed, but her facial rash has only partially resolved.

Dentists and specialists in oral medicine had important roles in early diagnosis in the present case. Early detection of disease was useful in managing the oral lesions, which were eventually successfully controlled. FAO $0.1 \%$ combined with systemic steroid use was reported to be effective in treating oral PV among Thai patients (8). Maintenance of oral hygiene was also very important in promoting lesion healing in the present case. At 8.5 years of follow-up, the patient shows some improvement and has better quality- of- life.

With the exception of PV, the present autoimmune diseases are uncommon connective tissue diseases. Disease development was slow but progressive in our patient and involved multiple autoimmune diseases. Although the pathogenesis of this disease is unclear, one report showed that abnormalities in tumor necrosis factor (TNF) were associated with dermatomyositis (9). Amyotrophic dermatomyositis occurs in a small subgroup of patients with dermatomyositis, who present only with the typical skin rash.
The present case was challenging because we could not predict or prevent progression of this rare disease, as the involvement of other autoimmune diseases makes such predictions difficult. If disease progression is observed, accurate treatment can be delivered. To our knowledge, there are no other case reports of oral PV followed by DLE and dermatomyositis. Our patient was very difficult to manage because of the lack of an established treatment. Close long-term follow-up of such rare cases is required because the disease may later affect other organs.

\section{Acknowledgments}

We express our thanks to Dr. Nop Porntrakulseree for taking the photographs of our patient. We also thank the Research Unit in Oral Diseases for their support and Dr. Kevin Tompkins for editing the manuscript. We are also grateful to the Faculty of Dentistry and Chulalongkorn University for their long-term support of our work. This case report was presented in an oral and poster presentation at the 11th Biennial Congress of the European Association of Oral Medicine in Athens, Greece, on 13-15 September 2012.

\section{References}

1. Black M, Mignogna MD, Scully C (2005) Number II. Pemphigus vulgaris. Oral Dis 11, 119-130.

2. Iamaroon $A$, Boonyawong $P$, Klanrit $P$, Prasongtunskul S, Thongprasom K (2006) Characterization of oral pemphigus vulgaris in Thai patients. J Oral Sci 48, 43-46.

3. Rodriguez DB (1981) Treatment for three ostomy patients with systemic skin disorders: psoriasis, pemphigus, and dermatomyositis. J Enterostomal Ther 8, 31-32.

4. Chow SK, Yeab SS (2000) Amyopathic dermatomyositis and pulmonary fibrosis. Clin Rheumatol 19, 484-485.

5. Narbutt J, Torzecka JD, Sysa-Jedrzejowska A, Pas HH (2003) Pemphigus foliaceus in an 11-year-old boy with dermatomyositis: simple coincidence or familial immunological background? Br J Dermatol 148, 838-839.

6. Black M, Marshman G (2011) Dermatomyositis and pemphigus vulgaris: association or coincidence? Australas $\mathrm{J}$ Dermatol 52, e11-14.

7. Calebotta A, Cirocco A, Giansante E, Reyes O (2004) Systemic lupus erythematosus and pemphigus vulgaris: association or coincidence. Lupus 13, 951-953.

8. Thongprasom K, Korkij W (2002) Fluocinolone acetonide $0.1 \%$ solution and prednisolone in the treatment of oral pemphigus: 12-year follow-up. Eastern J Med 7, 47.

9. Werth VP, Callen JP, Ang G, Sullivan KE (2002) Associations of tumor necrosis factor alpha and HLA polymorphisms with adult dermatomyositis: implications for a unique pathogenesis. J Invest Dermatol 119, 617-620. 